DOI: 10.17516/1997-1397-2020-13-3-285-296

УДК 517.55

\title{
On Some Examples of Systems of Transcendent Equations
}

\author{
Alexander M. Kytmanov* \\ Olga V. Khodos ${ }^{\dagger}$ \\ Siberian Federal University \\ Krasnoyarsk, Russian Federation
}

Received 06.02.2020, received in revised form 16.03.2020, accepted 09.04.2020

\begin{abstract}
This article discusses examples of transcendent systems of equations of a general form. The residue integrals are determined over the cycles associated with the system. Formulas are given for their calculation and their relationship with the power sums of the roots of the system is established.
\end{abstract}

Keywords: transcendent systems of equations, residue integrals, power sums of roots.

Citation: A.M.Kytmanov, O.V.Khodos, On some Examples of Systems of transcendent Equations, J. Sib. Fed. Univ. Math. Phys., 2020, 13(3), 285-296. DOI: 10.17516/1997-1397-2020-13-3-285-296.

For systems of nonlinear algebraic equations in $\mathbb{C}^{n}$, based on a multidimensional logarithmic residue, formulas were previously obtained for finding power sums of the roots of a system without calculating the roots themselves (see [1-3]). For different types of systems, such formulas have different forms. On this basis, a new method for the study of systems of algebraic equations in $\mathbb{C}^{n}$ is constructed. It arose in the work of L. A. Aizenberg [1], and its development is continued in monographs [2,4]. Its main idea is to find power sums of roots of systems (for positive powers) and then using one-dimensional or multidimensional recurrent Newton formulas (see [5]). Unlike the classical method of elimination, it is less labor intensive and does not increase the multiplicity of roots. It is based on a formula (see [1]) obtained using the multidimensional logarithmic residue, to find the sum of the values of an arbitrary polynomial in the roots of a given systems of algebraic equations without finding the roots themselves.

For systems of transcendent equations, formulas for the sum of the values of the roots of the system, as a rule, cannot be obtained, since the number of roots of a system can be infinite and a series of coordinates of such roots can be diverging. Nevertheless, transcendent systems of equations arise, for example, in the problems of chemical kinetics $[6,7]$. Thus, the urgent task is to consider such systems.

In the works [8-15] power sums of roots are considered for a negative power for different systems of non-algebraic (transcendent) equations. To compute these power sums, a residue integral is used, the integration of which is carried out over skeletons of polycircles centered at zero. Note that this residue integral is not, generally speaking, a multidimensional logarithmic residue or a Grothendieck residue. For various types of lower homogeneous systems of functions included in the system, formulas are given for finding residue integrals, their relationship with power sums of the roots of the system to a negative degree is established.

Article [16] investigated more complex systems in which the lower homogeneous parts are decomposed into linear factors and integration cycles in residue integrals, and are constructed from these factors.

In [15], a system is studied that arises in the Zel'dovich-Semenov model (see [6,7]) in chemical kinetics.

\footnotetext{
*AKytmanov@sfu-kras.ru

† khodos o@mail.ru

(C) Siberian Federal University. All rights reserved
} 
The object of this study is transcendent systems of equations in which the lower homogeneous parts of the functions included in the system form a non-degenerate system of algebraic equations: formulas are found for calculating the residue integrals, power sums of roots for a negative power, their relationship with the residue integrals is established. See [16,17].

Let $f_{1}(z), \ldots, f_{n}(z)$ be a system of functions holomorphic in a neighborhood of the origin in a multidimensional complex space $\mathbb{C}^{n}, z=\left(z_{1}, \ldots, z_{n}\right)$.

We expand functions $f_{1}(z), \ldots, f_{n}(z)$ into Taylor series in a neighborhood of the origin and consider a system of equations of the form

$$
f_{i}(z)=P_{i}(z)+Q_{i}(z)=0, \quad i=1, \ldots, n,
$$

where $P_{i}$ is the lower homogeneous part of the Taylor expansion of the function $f_{i}(z)$. The degree of all monomials (in the totality of variables) included in $P_{i}$, is $m_{i}, i=1, \ldots, n$. In functions $Q_{i}$, the degrees of all monomials are strictly greater than $m_{i}$.

The expansion of the functions $Q_{j}, P_{j}, j=1, \ldots, n$, in a neighborhood of zero in Taylor series converging absolutely and uniformly in this neighborhood has the form

$$
\begin{aligned}
Q_{j}(z) & =\sum_{\|\alpha\|>m_{j}} a_{\alpha}^{j} z^{\alpha}, \\
P_{j}(z) & =\sum_{\|\beta\|=m_{j}} b_{\beta}^{j} z^{\beta}, \\
j & =1, \ldots, n,
\end{aligned}
$$

where $\alpha=\left(\alpha_{1}, \ldots, \alpha_{n}\right), \beta=\left(\beta_{1}, \ldots, \beta_{n}\right)$ are multi-indices i.e. $\alpha_{j}$ and $\beta_{j}$ are non-negative integers, $j=1, \ldots, n,\|\alpha\|=\alpha_{1}+\ldots+\alpha_{n},\|\beta\|=\beta_{1}+\ldots+\beta_{n}$, and monomials $z^{\alpha}=z_{1}^{\alpha_{1}}$. $z_{2}^{\alpha_{2}} \cdots z_{n}^{\alpha_{n}}, z^{\beta}=z_{1}^{\beta_{1}} \cdot z_{2}^{\beta_{2}} \cdots z_{n}^{\beta_{n}}$.

In what follows, we will assume that the system of polynomials $P_{1}(z), \ldots, P_{n}(z)$ it is nondegenerate, i.e. its common zero is only the point 0 , the origin. Consider an open set (special analytic polyhedron) of the form

$$
D_{P}\left(r_{1}, \ldots, r_{n}\right)=\left\{z:\left|P_{i}(z)\right|<r_{i}, i=1, \ldots, n\right\},
$$

where $r_{1}, \ldots, r_{n}$ are positive numbers. Its skeleton has the form

$$
\Gamma_{P}\left(r_{1}, \ldots, r_{n}\right)=\Gamma_{P}(r)=\left\{z:\left|P_{i}(z)\right|=r_{i}, i=1, \ldots, n\right\} .
$$

These sets play an important role in the theory of multidimensional residues (see, for example, [2]).

For sufficiently small $r_{i}$, the cycles $\Gamma_{P}$ lie in the domain of holomorphy of functions $f_{i}$, therefore, the series

$$
\sum_{\|\alpha\|>m_{i}}\left|a_{\alpha}^{j}\right| r_{1}^{\alpha_{1}} \cdots r_{n}^{\alpha_{n}}
$$

converge, $i=1,2, \ldots, n$. Then on the cycle $\Gamma_{P}(t r)=\Gamma_{P}\left(t r_{1}, t r_{2}, \ldots, t r_{n}\right)$ for sufficiently small $t>0$, we have

$$
\left|P_{i}(t r)\right|=\left|\sum_{\|\beta\|=m_{i}} b_{\beta}^{i}(t r)^{\beta}\right|=\sum_{\|\beta\|=m_{i}} t^{\|\beta\|}\left|b_{\beta}^{i}\right| r^{\beta}=t^{m_{i}} \sum_{\|\beta\|=m_{i}}\left|b_{\beta}^{i}\right| r^{\beta}, \quad i=1, \ldots, n,
$$

and

$$
\left|Q_{i}(t r)\right|=\left|\sum_{\|\alpha\|>m_{i}} a_{\alpha}^{i}(t r)^{\alpha}\right| \leqslant \sum_{\|\alpha\|>m_{i}} t^{\|\alpha\|}\left|a_{\alpha}^{i}\right| r^{\alpha}=t^{m_{i}+1} \sum_{\|\alpha\|>m_{i}}\left|a_{\alpha}^{i}\right| r^{\alpha} t^{\|\alpha\|-\left(m_{i}+1\right)}
$$


Therefore, for sufficiently small $t$ on the cycle $\Gamma_{P}(t r)$ the inequalities hold

$$
\left|P_{i}(z)\right|>\left|Q_{i}(z)\right|, \quad i=1,2, \ldots, n .
$$

Thus,

$$
f_{i}(z) \neq 0 \quad \text { на } \quad \Gamma_{P}(t r), \quad i=1,2, \ldots, n .
$$

In what follows, we assume that $t=1$, that is, that the inequality (4) is valid on the cycle $\Gamma_{P}\left(r_{1}, \ldots, r_{n}\right)$.

We introduce the concept of residue integral $J_{\gamma}$ (see [18]). Define

$$
\begin{gathered}
J_{\gamma}=\frac{1}{(2 \pi \sqrt{-1})^{n}} \int_{\Gamma_{P}} \frac{1}{z^{\gamma+1}} \cdot \frac{d f}{f}= \\
=\frac{1}{(2 \pi \sqrt{-1})^{n}} \int_{\Gamma_{P}} \frac{1}{z_{1}^{\gamma_{1}+1} \cdot z_{2}^{\gamma_{2}+1} \ldots z_{n}^{\gamma_{n}+1}} \cdot \frac{d f_{1}}{f_{1}} \wedge \frac{d f_{2}}{f_{2}} \wedge \ldots \wedge \frac{d f_{n}}{f_{n}},
\end{gathered}
$$

where $\gamma=\left(\gamma_{1}, \ldots \gamma_{n}\right)$ is a multi-index. This residue integral is defined if $r_{1}, \ldots, r_{n}$ is chosen so that the inequality (4) holds and the cycle $\Gamma_{P}$ does not intersect with the coordinate planes. Note that this integral is not a multidimensional logarithmic residue or a Grothendieck residue.

Recall some concepts from the space of the theory of functions $\overline{\mathbb{C}}^{n}$ which equal to the product of $n$ copies of Riemann spheres $\mathbb{C P}^{1}$, i.e. $\overline{\mathbb{C}}^{n}=\mathbb{C P}^{1} \times \cdots \times \mathbb{C P}^{1}$.

Let $z_{j}: w_{j}$ be homogeneous coordinates in the $j$-th factor of the space $\overline{\mathbb{C}}^{n}$ and let

$$
F_{j}\left(z_{1}, w_{1}, \ldots, z_{n}, w_{n}\right)=0, \quad j=1, \ldots, n
$$

be a system of equations consisting of polynomials $F_{j}$ homogeneous for each pair of variables $\left(z_{k}, w_{k}\right), k=1, \ldots, n$. We will consider only those roots $\left(z_{1}, w_{1}, \ldots, z_{n}, w_{n}\right)$ systems (6) for which

$$
\left(z_{k}, w_{k}\right) \in \mathbb{C}^{2} \backslash\{(0,0)\}, \quad k=1, \ldots, n .
$$

The roots of the system (6) with pairs having proportional coordinates determine one root $\left(z_{1}: w_{1}, \ldots, z_{n}: w_{n}\right)$ in $\overline{\mathbb{C}}^{n}$.

Let

$$
a=\left(z_{1}^{(0)}: w_{1}^{(0)}, \ldots, z_{n}^{(0)}: w_{n}^{(0)}\right)
$$

be the root of the system (6) for which all $w_{k}^{(0)} n e q 0$. Then the point $\left(z_{1}, 1, \ldots, z_{n}, 1\right)$ is the root of the system

$$
F_{j}\left(z_{1}, 1, \ldots, z_{n}, 1\right)=0, \quad j=1, \ldots, n,
$$

in $\mathbb{C}^{n}$. Roots of $a$ for which some $w_{j}^{(0)}$ are equal to zero correspond to infinitely remote roots in $\overline{\mathbb{C}}^{n}$.

For a given system of equations of the form (1) for which all $f_{j}(z)$ are polynomials, then in order to find the infinitely remote roots of this system in $\overline{\mathbb{C}}^{n}$, you must first go to homogeneous coordinates, substituting the $z_{k} / w_{k}$ relationship instead of $z_{k}$ and discarding the resulting denominator, thereby obtaining a system of type (6). Solving it, we find ordinary roots and infinitely remote roots of the system (1).

We return to the consideration of the system (1). Assume that, in addition to non-degeneracy, the system $P_{1}(z), \ldots, P_{n}(z)$ does not have infinite roots in the space $\overline{\mathbb{C}}^{n}$.

We now consider as functions $Q_{i}(z), i=1, \ldots, n$, polynomials of the form

$$
Q_{i}(z)=\sum_{\|\alpha\|>m_{i}} a_{\alpha}^{i} z^{\alpha}
$$


Suppose that for each $i$-th equation in (1) the conditions

$$
\operatorname{deg}_{z_{i}} P_{i}<\operatorname{deg}_{z_{i}} Q_{i}, \quad \operatorname{deg}_{z_{j}} P_{i} \geqslant \operatorname{deg}_{z_{j}} Q_{i}, \quad j \neq i .
$$

Here $\operatorname{deg}_{z_{i}} P(z)$ is the degree of the polynomial $P$ in the variable $z_{i}$ for the remaining variables We have $\operatorname{deg} P_{i}=m_{i}$. Denote $\operatorname{deg} Q_{i}=s_{i}, \operatorname{a~deg}_{z_{j}} P_{i}=m_{i}^{j}, \quad \operatorname{deg}_{z_{j}} Q_{i}=s_{i}^{j}$. Then $m_{i}<s_{i}$, $m_{i}^{i}<s_{i}^{i}, i=1, \ldots, n$. In addition, $m_{i}^{j} \geqslant s_{i}^{j}$ for $j \neq i$. Cases when $\sum_{j=1}^{n} m_{i}^{j}>m_{i}$.

In all functions, we write $f_{i}(z)=P_{i}(z)+Q_{i}(z), i=1,2, \ldots, n$, and replace $z_{i}=\frac{1}{w_{i}}, i=$ $1, \ldots, n$, assuming that all $w_{i} \neq 0$. We get

$$
P_{i}\left(\frac{1}{w_{1}}, \ldots, \frac{1}{w_{n}}\right)=\sum_{\|\beta\|=m_{i}} b_{\beta}^{i} \frac{1}{w_{1}^{\beta_{1}}} \cdots \frac{1}{w_{n}^{\beta_{n}}}=\frac{1}{w_{1}^{m_{i}^{1}}} \cdots \frac{1}{w_{n}^{m_{i}^{n}}} \sum_{\| \beta \mid=m_{i}} b_{\beta}^{i} w_{1}^{m_{i}^{1}-\beta_{1}} \cdots w_{n}^{m_{i}^{n}-\beta_{n}},
$$

and

$$
Q_{i}\left(\frac{1}{w_{1}}, \ldots, \frac{1}{w_{n}}\right)=\sum_{\|\alpha\|>m_{i}} a_{\alpha}^{i} \frac{1}{w_{1}^{\alpha_{1}}} \cdots \frac{1}{w_{n}^{\alpha_{n}}}=\frac{1}{w_{1}^{s_{i}^{1}}} \cdots \frac{1}{w_{n}^{s_{n}^{n}}} \sum_{\|\alpha\|>m_{i}} a_{\alpha}^{i} w_{1}^{s_{i}^{1}-\alpha_{1}} \cdots w_{n}^{s_{i}^{n}-\alpha_{n}}
$$

We have

$$
\begin{aligned}
f_{i}\left(\frac{1}{w_{1}}, \ldots, \frac{1}{w_{n}}\right) & =P_{i}\left(\frac{1}{w_{1}}, \ldots, \frac{1}{w_{n}}\right)+Q_{i}\left(\frac{1}{w_{1}}, \ldots, \frac{1}{w_{n}}\right)= \\
& =\frac{1}{w_{1}^{m_{i}^{1}} \cdots w_{i}^{s_{i}^{i}} \cdots w_{n}^{m_{i}^{n}}} \cdot\left(\tilde{P}_{i}(w)+\tilde{Q}_{i}(w)\right),
\end{aligned}
$$

where $\tilde{P}_{i}$ are homogeneous polynomials

$$
\begin{gathered}
\tilde{P}_{i}\left(w_{1}, \ldots, w_{n}\right)=w_{1}^{m_{i}^{1}} \cdots w_{i}^{s_{i}^{i}} \cdots w_{n}^{m_{i}^{n}} \cdot P_{i}\left(\frac{1}{w_{1}}, \ldots, \frac{1}{w_{n}}\right)= \\
=w_{i}^{s_{i}^{i}-m_{i}^{i}} \sum_{\|\beta\|=m_{i}} b_{\beta}^{i} w_{1}^{m_{i}^{1}-\beta_{1}} \cdots w_{n}^{m_{i}^{n}-\beta_{n}}=w_{i}^{s_{i}^{i}-m_{i}^{i}} \cdot \tilde{\tilde{P}}_{i},
\end{gathered}
$$

and $\tilde{\tilde{P}}_{i}$ are homogeneous polynomials

$$
\tilde{\tilde{P}}_{i}=\sum_{\|\beta\|=m_{i}} b_{\beta}^{i} w_{1}^{m_{i}^{1}-\beta_{1}} \cdot \ldots \cdot w_{n}^{m_{i}^{n}-\beta_{n}} .
$$

In $\tilde{\tilde{P}}_{i}$, neither $w_{1}, \ldots$, nor $w_{n}$.

The polynomials $\tilde{Q}_{i}$ have the form

$$
\begin{gathered}
\quad \tilde{Q}_{i}\left(w_{1}, \ldots, w_{n}\right)=w_{1}^{m_{i}^{1}} \cdots w_{i}^{s_{i}^{i}} \cdots w_{n}^{m_{i}^{n}} \cdot Q_{i}\left(\frac{1}{w}, \ldots, \frac{1}{w_{n}}\right)= \\
=w_{1}^{m_{i}^{1}} \cdots w_{i}^{s_{i}^{i}} \cdots w_{n}^{m_{i}^{n}} \cdot \frac{1}{w_{1}^{s_{i}^{1}}} \cdots \frac{1}{w_{n}^{s_{i}^{n}}} \sum_{\|\alpha\|>m_{i}} a_{\alpha}^{i} w_{1}^{s_{i}^{1}-\alpha_{1}} \cdots w_{n}^{s_{i}^{n}-\alpha_{n}}= \\
=w_{1}^{m_{i}^{1}-s_{i}^{1}} \cdots\left[w_{i}\right] \cdots w_{n}^{m_{i}^{n}-s_{i}^{n}} \cdot \sum_{\|\alpha\|>m_{i}} a_{\alpha}^{i} w_{1}^{m_{i}^{1}-\alpha_{1}} \cdots w_{n}^{m_{i}^{n}-\alpha_{n}} .
\end{gathered}
$$


Denote by $\tilde{f}_{i}$ the functions

$$
\tilde{f}_{i}(w)=\tilde{P}_{i}(w)+\tilde{Q}_{i}(w)=w_{i}^{s_{i}^{i}-m_{i}^{i}} \cdot \tilde{\tilde{P}}_{i}+\tilde{Q}_{i}(w), \quad i=1,2, \ldots, n
$$

We have

$$
\operatorname{deg} \tilde{P}_{i}>\operatorname{deg} \tilde{Q}_{i}, \quad i=1, \ldots, n .
$$

Consider a system of equations of the form (1) with polynomials $Q_{i}(z)$ satisfying the conditions (8).

Let $\Gamma_{\tilde{P}}=\Gamma_{\tilde{P}}(\varepsilon)$ denote the cycle

$$
\Gamma_{\tilde{P}}=\left\{w \in \mathbb{C}^{n}:\left|\tilde{P}_{i}\right|=\varepsilon_{i}, \quad \varepsilon_{i}>0, i=1, \ldots, n\right\} .
$$

This cycle does not intersect with the coordinate planes for almost all $\varepsilon_{i}, i=1, \ldots, n$.

Consider the residue integral $\tilde{J}_{\gamma}$ of the form

$$
\tilde{J}_{\gamma}=\frac{1}{(2 \pi \sqrt{-1})^{n}} \int_{\Gamma_{\tilde{P}}} w^{\gamma+I} \frac{d f(1 / w)}{f(1 / w)},
$$

where $w^{\gamma+I}=w_{1}^{\gamma_{1}+1} \cdots w_{n}^{\gamma_{n}+1}, f(1 / w)=f_{1}\left(1 / w_{1}, \ldots, 1 / w_{n}\right) \cdots f_{n}\left(1 / w_{1}, \ldots, 1 / w_{n}\right), d f(1 / w)=$ $=d f_{1}\left(1 / w_{1}, \ldots, 1 / w_{n}\right) \wedge \ldots \wedge d f_{n}\left(1 / w_{1}, \ldots, 1 / w_{n}\right)$.

In fact, $\tilde{J}_{\gamma}$ is obtained from the integral $J_{\gamma}(5)$ using the substitution in the integrand $z_{j}=$ $1 / w_{j}, j=1, \ldots, n$, and replacing $\Gamma_{P}$ by $\Gamma_{\tilde{P}}$. But the equality of these integrals needs to be proved.

Since the inequalities (11) hold for functions from the system (10), and the system of functions $\tilde{P}_{1}(w), \ldots, \tilde{P}_{n}(w)$ is non-degenerate, the well known Bezout theorem says that the system of equations

$$
\tilde{f}_{j}(w)=0, \quad j=1, \ldots, n,
$$

has a finite number of roots (counting each root so many times what its multiplicity is) and this number is equal to the product of the degrees of the polynomials $\tilde{P}_{j}(w)$.

We cite the theorem from [16].

Theorem 1. The following equality holds:

$$
\begin{gathered}
\sum_{j=1}^{p} \frac{1}{z_{j 1}^{\gamma_{1}+1} \cdot z_{j 2}^{\gamma_{2}+1} \cdots z_{j n}^{\gamma_{n}+1}}= \\
=\sum_{\|\alpha\| \leqslant\|\gamma\|+n}(-1)^{\|\alpha\|} \int_{\Gamma_{\tilde{P}}}\left[\tilde{\Delta} \cdot w_{1}^{\gamma_{1}+1} \cdot w_{2}^{\gamma_{2}+1} \cdots w_{n}^{\gamma_{n}+1} \cdot \frac{\tilde{Q}_{1}^{\alpha_{1}} \cdot \tilde{Q}_{2}^{\alpha_{2}} \cdots \tilde{Q}_{n}^{\alpha_{n}}}{\tilde{P}_{1}^{\alpha_{1}+1} \cdot \tilde{P}_{2}^{\alpha_{2}+1} \cdots \tilde{P}_{n}^{\alpha_{n}+1}}\right] d w,
\end{gathered}
$$

where $\tilde{\Delta}$ is the Jacobian of the system (10).

For what follows, we need a generalized Grothendieck residue transformation formula (see [19], as well as $[4, \mathrm{Ch} .2])$.

Theorem 2 ([19]). Let $h(w)$ be a holomorphic function, and the polynomials $f_{k}(w)$ and $g_{j}(w)$, $j, k=1, \ldots, n$, be related by

$$
g_{j}=\sum_{k=1}^{n} a_{j k} f_{k}, \quad j=1,2, \ldots, n,
$$

the matrix $A=\left\|a_{j k}\right\|_{j, k=1}^{n}$ consists of polynomials. Let us consider cycles

$$
\Gamma_{f}=\left\{w:\left|f_{j}(w)\right|=r_{j}, j=1, \ldots, n\right\}, \quad \Gamma_{g}=\left\{w:\left|g_{j}(z)\right|=r_{j}, j=1, \ldots, n\right\},
$$


where all $r_{j}>0$.

Then the equality is valid:

$$
\int_{\Gamma_{f}} h(w) \frac{d w}{f^{\alpha}}=\sum_{K, \sum_{s=1}^{n} k_{s j}=\beta_{s}} \frac{\beta !}{\prod_{s, j=1}^{n}\left(k_{s j}\right) !} \int_{\Gamma_{g}} h(w) \frac{\operatorname{det} A \prod_{s, j=1}^{n} a_{s j}^{k_{s j}} d w}{g^{\beta}},
$$

where $\beta !=\beta_{1} ! \beta_{2} ! \ldots \beta_{n}, \beta=\left(\beta_{1}, \beta_{2}, \ldots, \beta_{n}\right)$, the summation in the formula is over all integer non-negative matrices $K=\left\|k_{s j}\right\|_{s, j=1}^{n}$ with the conditions that the sum $\sum_{s=1}^{n} k_{s j}=\alpha_{j}$, then $\beta_{j}=$ $=\sum_{j=1}^{n} k_{j s}$

Here $f^{\alpha}=f_{1}^{\alpha_{1}} \cdots f_{n}^{\alpha_{n}}, g^{\beta}=g_{1}^{\beta_{1}} \cdots g_{n}^{\beta_{n}}$.

From this theorem, a statement is obtained in [16].

Theorem 3. The formulas are valid

$$
\begin{gathered}
\sum_{j=1}^{p} \frac{1}{z_{j 1}^{\gamma_{1}+1} \cdot z_{j 2}^{\gamma_{2}+1} \cdots z_{j n}^{\gamma_{n}+1}}=\frac{(-1)^{n}}{(2 \pi \sqrt{-1})^{n}} \int_{\Gamma_{\tilde{P}}} w_{1}^{\gamma_{1}+1} \cdot w_{2}^{\gamma_{2}+1} \cdots w_{n}^{\gamma_{n}+1} \cdot \frac{d \tilde{f}_{1}}{\tilde{f}_{1}} \wedge \frac{d \tilde{f}_{2}}{\tilde{f}_{2}} \wedge \ldots \wedge \frac{d \tilde{f}_{n}}{\tilde{f}_{n}}= \\
=\sum_{\|\alpha\| \leqslant\|\gamma\|+n} \frac{(-1)^{n+\|\alpha\|}}{(2 \pi \sqrt{-1})^{n}} \int_{\Gamma_{\tilde{P}}} w_{1}^{\gamma_{1}+1} \cdot w_{2}^{\gamma_{2}+1} \cdots w_{n}^{\gamma_{n}+1} \cdot \frac{\tilde{\Delta} \cdot \tilde{Q}_{1}^{\alpha_{1}} \cdot \tilde{Q}_{2}^{\alpha_{2}} \cdots \tilde{Q}_{n}^{\alpha_{n}} d w_{1} \wedge d w_{2} \wedge \ldots \wedge d w_{n}}{\tilde{P}_{1}^{\alpha_{1}+1} \cdot \tilde{P}_{2}^{\alpha_{2}+1} \cdots \tilde{P}_{n}^{\alpha_{n}+1}}= \\
=\sum_{\|K\| \leqslant\|\gamma\|+n} \frac{(-1)^{\|K\|+n} \prod_{s=1}^{n}\left(\sum_{j=1}^{n} k_{s j}\right) !}{\prod_{s, j=1}^{n}\left(k_{s j}\right) !} \mathfrak{M}\left[\frac{w^{\gamma+I} \cdot \tilde{\Delta} \cdot \operatorname{det} A \cdot Q^{\alpha} \prod_{s, j=1}^{n} a_{s j}^{k_{s j}}}{\prod_{j=1}^{n} w_{j}^{\beta_{j} N_{j}+\beta_{j}+N_{j}}}\right],
\end{gathered}
$$

where $\|K\|=\sum_{s, j=1}^{n} k_{s j}$, and the functional $\mathfrak{M}$ maps the Laurent polynomial to its free term.

In fact, in Theorem 3, analogues of the classical Waring formulas for finding power sums of the roots of a system of algebraic equations are obtained.

Note that in [20] general algebraic systems of equations were considered, decompositions of their solutions in hypergeometric series were obtained. In addition, it proves analogues of Waring's formulas for systems of the form

$$
y_{j}^{m_{j}}+\sum_{\lambda \in \Lambda^{(j)} \cup\{0\}} x_{\lambda}^{(j)} y^{\lambda}=0, \quad \lambda_{1}+\ldots+\lambda_{n}<m_{j}, \quad j=1, \ldots, n,
$$

those higher homogeneous parts are monomials. We considered other (more general) systems of equations with functions of the form (10).

Consider a more general situation. Let the functions $f_{j}$ be meromorphic and have the form

$$
f_{j}(z)=\frac{f_{j}^{(1)}(z)}{f_{j}^{(2)}(z)}, \quad j=1,2, \ldots, n,
$$

where $f_{j}^{(1)}(z)$ and $f_{j}^{(2)}(z)$ are entire functions in $\mathbb{C}^{n}$ that decompose into infinite products uniformly converging in $\mathbb{C}^{n}, f_{j}^{(2)}(0) \neq 0$,

$$
f_{j}^{(1)}(z)=\prod_{s=1}^{\infty} f_{j, s}^{(1)}(z), \quad f_{j}^{(2)}(z)=\prod_{s=1}^{\infty} f_{j, s}^{(2)}(z),
$$


moreover, each of the factors has the form $P_{j, s}(z)+Q_{j, s}(z)$, and $Q_{j, s}(z)$ satisfy conditions $(8)$, $s=1,2, \ldots$.

For each set of indices $j_{1}, \ldots, j_{n}$, where $j_{1}, \ldots, j_{n} \in \mathbb{N}$, and each set of numbers $i_{1}, \ldots, i_{n}$, where $i_{1}, \ldots, i_{n}$ are equal 1 or 2 , systems of nonlinear equations

$$
f_{1, j_{1}}^{\left(i_{1}\right)}(z)=0, \quad f_{2, j_{2}}^{\left(i_{2}\right)}(z)=0, \ldots, f_{n, j_{n}}^{\left(i_{n}\right)}(z)=0
$$

have a finite number of roots not lying on coordinate planes.

The roots of all such systems (not lying on the coordinate planes) are no more than a countable set. Renumber them (taking into account multiplicities):

$$
z_{(1)}, z_{(2)}, \ldots, z_{(l)}, \ldots
$$

Denote by $\sigma_{\beta+I}$ the expression

$$
\sigma_{\beta+I}=\sum_{l=1}^{\infty} \frac{\varepsilon_{l}}{z_{1(l)}^{\beta_{1}+1} \cdot z_{2(l)}^{\beta_{2}+1} \cdots z_{n(l)}^{\beta_{n}+1}} .
$$

Here $\beta_{1}, \ldots, \beta_{n}$, as before, are non-negative integers, and the sign $\varepsilon_{l}$ is +1 , if in a system of the form (16), the root which is $z_{(l)}$, includes an even number of functions $f_{j_{s}}^{(2)}$; and is equal to -1 if in a system of the form (16), the root which is $z_{(l)}$, includes an odd number of functions $f_{j_{s}}^{(2)}$.

For a system (16) composed of functions of the form (15), the points $z_{(l)}$ are roots or singular points (poles). All functions $f_{j}$ are holomorphic in a neighborhood of zero and are defined for them integrals $J_{\beta}$, since they have the form (1).

Theorem 4. For a system of equations with meromorphic functions (15) the series (17) absolutely converges, and

$$
J_{\beta}=(-1)^{n} \sigma_{\beta+I} .
$$

\section{Example 1.}

Consider a system of equations in two complex variables

$$
\left\{\begin{array}{l}
f_{1}\left(z_{1}, z_{2}\right)=z_{1}-z_{2}+a z_{1}^{2}+b z_{1}^{3}=0 \\
f_{2}\left(z_{1}, z_{2}\right)=1+c z_{2}=0
\end{array}\right.
$$

We make the change of variables $z_{1}=\frac{1}{w_{1}}, z_{2}=\frac{1}{w_{2}}$. Our system will take the form

$$
\left\{\begin{array}{l}
\widetilde{f}_{1}=w_{1}^{2} w_{2}-w_{1}^{3}+a w_{1} w_{2}+b w_{2}=0 \\
\widetilde{f}_{2}=w_{2}+c=0
\end{array}\right.
$$

The Jacobian of the system (18) $\widetilde{\Delta}$ is

$$
\widetilde{\Delta}=\left|\begin{array}{cc}
2 w_{1} w_{2}-3 w_{1}^{2}+a w_{2} & w_{1}^{2}+a w_{1}+b \\
0 & 1
\end{array}\right|=2 w_{1} w_{2}-3 w_{1}^{2}+a w_{2} .
$$

It is clear that

$$
\begin{gathered}
\left\{\begin{array}{l}
\widetilde{Q}_{1}=a w_{1} w_{2}+b w_{2}, \\
\widetilde{Q}_{2}=c .
\end{array}\right. \\
\left\{\begin{array}{l}
\widetilde{P}_{1}=w_{1}^{2} w_{2}-w_{1}^{3}, \\
\widetilde{P}_{2}=w_{2} .
\end{array}\right.
\end{gathered}
$$


Since

$$
\begin{aligned}
& w_{1}^{3}=a_{11} \widetilde{P}_{1}+a_{12} \widetilde{P}_{2}, \\
& w_{2}=a_{21} \widetilde{P}_{1}+a_{22} \widetilde{P}_{2},
\end{aligned}
$$

it is easy to show that the elements $a_{i j}$ of the matrix $A$ are equal

$$
\begin{gathered}
a_{11}=-1, a_{12}=w_{1}^{2}, \\
a_{21}=0, a_{22}=1 .
\end{gathered}
$$

Thus, $\operatorname{det} A=-1$.

By Theorem 3

$$
\begin{gathered}
J_{(0,0)}=\sum_{\|K\|=k_{11}+k_{12}+k_{21}+k_{22} \leqslant 2} \frac{(-1)^{\|K\|} \cdot\left(k_{11}+k_{12}\right) ! \cdot\left(k_{21}+k_{22}\right) !}{k_{11} ! \cdot k_{12} ! \cdot k_{21} ! \cdot k_{22} !} \times \\
\times \mathfrak{M}\left[\frac{\left(3 w_{1}^{2}-2 w_{1} w_{2}-a w_{2}\right) \cdot\left(a w_{1} w_{2}+b w_{2}\right)^{k_{11}+k_{21}} \cdot c^{k_{12}+k_{22}} \cdot(-1)^{k_{11}} \cdot\left(w_{1}^{2}\right)^{k_{12}} \cdot 0^{k_{21}} \cdot 1^{k_{22}}}{w_{1}^{3\left(k_{11}+k_{12}\right)+1} \cdot w_{2}^{\left(k_{21}+k_{22}\right)-1}}\right] .
\end{gathered}
$$

Simple calculations give that

$$
J_{(0,0)}=c^{2} .
$$

Recall the well-known decomposition of the sine function into an infinite product:

$$
\frac{\sin z}{z}=\prod_{k=1}^{\infty}\left(1-\frac{z^{2}}{k^{2} \pi^{2}}\right)
$$

which uniformly and absolutely converge on the complex plane and has a growth order of 1 .

Consider the system of equations

$$
\left\{\begin{array}{l}
f_{1}\left(z_{1}, z_{2}\right)=z_{1}-z_{2}+a z_{1}^{2}+b z_{1}^{3}=0 \\
f_{2}\left(z_{1}, z_{2}\right)=\frac{\sin z_{2}}{z_{2}}=0
\end{array}\right.
$$

Using the formula obtained above and the known sum, we obtain that the integral $J_{(0,0)}$ is equal to the sum of the series

$$
J_{(0,0)}=2 \sum_{s=1}^{\infty} \frac{1}{\pi^{2} s^{2}}=\frac{1}{3}
$$

Example 2. Consider a system of equations in two complex variables

$$
\left\{\begin{array}{l}
f_{1}\left(z_{1}, z_{2}\right)=z_{1} z_{2}+b_{1} z_{1}+b_{2} z_{2}=0 \\
f_{2}\left(z_{1}, z_{2}\right)=1+a_{1} z_{1}+a_{2} z_{2}=0
\end{array}\right.
$$

We make the change of variables $z_{1}=\frac{1}{w_{1}}, z_{2}=\frac{1}{w_{2}}$. Our system will take the form

$$
\left\{\begin{array}{l}
\widetilde{f}_{1}=1+b_{2} w_{1}+b_{1} w_{2}=0 \\
\widetilde{f}_{2}=w_{1} w_{2}+a_{2} w_{1}+a_{1} w_{2}=0
\end{array}\right.
$$

The Jacobian of the system $(24) \widetilde{\Delta}$ is

$$
\widetilde{\Delta}=\left|\begin{array}{cc}
b_{2} & b_{1} \\
w_{2}+a_{2} & w_{1}+a_{1}
\end{array}\right|=b_{2} w_{1}-b_{1} w_{2}+\left(a_{1} b_{2}-a_{2} b_{1}\right) .
$$


Notice that

$$
\begin{aligned}
& \left\{\begin{array}{l}
\widetilde{Q}_{1}=1, \\
\widetilde{Q}_{2}=a_{1} w_{2}+a_{2} w_{1} .
\end{array}\right. \\
& \left\{\begin{array}{l}
\widetilde{P}_{1}=b_{1} w_{2}+b_{2} w_{1}, \\
\widetilde{P}_{2}=w_{1} w_{2} .
\end{array}\right.
\end{aligned}
$$

We calculate $\operatorname{det} A$ :

Since

$$
\begin{aligned}
& w_{1}^{2}=a_{11} \widetilde{P}_{1}+a_{12} \widetilde{P}_{2}, \\
& w_{2}^{2}=a_{21} \widetilde{P}_{1}+a_{22} \widetilde{P}_{2},
\end{aligned}
$$

where $\widetilde{P}_{1}=b_{1} w_{2}+b_{2} w_{1}, \widetilde{P}_{2}=w_{1} w_{2}$.

Therefore, the elements of $a_{i i}$ are equal

$$
\begin{aligned}
& a_{11}=\frac{w_{1}}{b_{2}}, a_{12}=-\frac{b_{1}}{b_{2}}, \\
& a_{21}=\frac{w_{2}}{b_{1}}, a_{22}=-\frac{b_{2}}{b_{1}} .
\end{aligned}
$$

Hence,

$$
\operatorname{det} A=\frac{w_{2}}{b_{2}}-\frac{w_{1}}{b_{1}}=\frac{w_{2} b_{1}-w_{1} b_{2}}{b_{1} b_{2}} .
$$

Notice that

$$
\tilde{Q}_{1}=1, \quad \tilde{Q}_{2}=1 .
$$

Carrying out the same calculations as in the previous example, we obtain

$$
J_{(0,0)}=-\frac{2\left(a_{1}+b_{2}\right)}{\bar{\Delta}} .
$$

\section{Example 3.}

Consider a system of equations in two complex variables

$$
\left\{\begin{array}{l}
f_{1}\left(z_{1}, z_{2}\right)=a_{1} z_{1}-a_{2} z_{2}+z_{1}^{2}=0 \\
f_{2}\left(z_{1}, z_{2}\right)=b_{1} z_{1}+b_{2} z_{2}+z_{2}^{2}=0
\end{array}\right.
$$

It satisfies the conditions $(8)$ on $Q_{j}(z)$. We assume that $a_{1} b_{2}+a_{2} b_{1} \neq 0$, i.e. the system of lower homogeneous polynomials is non-degenerate.

We make the change of variables $z_{1}=\frac{1}{w_{1}}, z_{2}=\frac{1}{w_{2}}$. Our system will take the form

$$
\left\{\begin{array}{l}
\tilde{f}_{1}=-a_{2} w_{1}^{2}+a_{1} w_{1} w_{2}+w_{2}=0 \\
\tilde{f}_{2}=b_{2} w_{1} w_{2}+b_{1} w_{2}^{2}+w_{1}=0
\end{array}\right.
$$

This system has 4 roots, on the coordinate planes there is one root, $(0,0)$.

The Jacobian $\tilde{\Delta}$ of the system (24) is equal to

$$
\tilde{\Delta}=\left|\begin{array}{cc}
-2 a_{2} w_{1}+a_{1} w_{2} & a_{1} w_{1}+1 \\
b_{2} w_{2}+1 & 2 b_{1} w_{2}+b_{2} w_{1}
\end{array}\right|=-2 a_{2} b_{2} w_{1}^{2}-4 a_{2} b_{1} w_{1} w_{2}+2 a_{1} b_{1} w_{2}^{2}-a_{1} w_{1}-b_{2} w_{2}-1
$$

Notice that

$$
\tilde{Q}_{1}=w_{2}, \quad \tilde{Q}_{2}=w_{1}
$$




$$
\tilde{P}_{1}=-a_{2} w_{1}^{2}+a_{1} w_{1} w_{2}, \quad \tilde{P}_{2}=b_{2} w_{1} w_{2}+b_{1} w_{2}^{2} .
$$

To find the matrix $A$, we use Example 8.3 from [4].

We introduce the matrix

$$
\operatorname{Res}=\left(\begin{array}{cccc}
-a_{2} & a_{1} & 0 & 0 \\
0 & -a_{2} & a_{1} & 0 \\
0 & b_{2} & b_{1} & 0 \\
0 & 0 & b_{2} & b_{1}
\end{array}\right)
$$

The determinant $\Delta$ of the matrix Res is equal to $\Delta=a_{2} b_{1}\left(a_{2} b_{1}+a_{1} b_{2}\right)$.

We calculate some minors according to Example 8.3 from [4]:

$$
\begin{aligned}
& \tilde{\Delta}_{1}=\left|\begin{array}{ccc}
-a_{2} & a_{1} & 0 \\
b_{2} & b_{1} & 0 \\
0 & b_{2} & b_{1}
\end{array}\right|=-a_{2} b_{1}^{2}-a_{1} b_{1} b_{2}, \quad \tilde{\Delta}_{2}=-\left|\begin{array}{ccc}
a_{1} & 0 & 0 \\
b_{2} & b_{1} & 0 \\
0 & b_{2} & b_{1}
\end{array}\right|=-a_{1} b_{1}^{2}, \\
& \widetilde{\Delta}_{3}=\left|\begin{array}{ccc}
a_{1} & 0 & 0 \\
-a_{2} & a_{1} & 0 \\
0 & b_{2} & b_{1}
\end{array}\right|=a_{1}^{2} b_{1}, \quad \tilde{\Delta}_{4}=-\left|\begin{array}{ccc}
a_{1} & 0 & 0 \\
-a_{2} & a_{1} & 0 \\
b_{2} & b_{1} & 0
\end{array}\right|=0 . \\
& \Delta_{1}=-\left|\begin{array}{ccc}
0 & -a_{2} & a_{1} \\
0 & b_{2} & b_{1} \\
0 & 0 & b_{2}
\end{array}\right|=0, \quad \Delta_{2}=\left|\begin{array}{ccc}
-a_{2} & a_{1} & 0 \\
0 & b_{2} & b_{1} \\
0 & 0 & b_{2}
\end{array}\right|=-a_{2} b_{2}^{2}, \\
& \Delta_{3}=-\left|\begin{array}{ccc}
-a_{2} & a_{1} & 0 \\
0 & -a_{2} & a_{1} \\
0 & 0 & b_{2}
\end{array}\right|=-a_{2}^{2} b_{2}, \quad \Delta_{4}=\left|\begin{array}{ccc}
-a_{2} & a_{1} & 0 \\
0 & -a_{2} & a_{1} \\
0 & b_{2} & b_{1}
\end{array}\right|=a_{2}^{2} b_{1}+a_{1} a_{2} b_{2} .
\end{aligned}
$$

Therefore, the elements $a_{i j}$ of the matrix $A$ are equal

$$
\begin{gathered}
a_{11}=\frac{1}{\Delta}\left(\tilde{\Delta}_{1} w_{1}+\tilde{\Delta}_{2} w_{2}\right)=\frac{1}{\Delta}\left(\left(-a_{2} b_{1}^{2}-a_{1} b_{1} b_{2}\right) w_{1}-a_{1} b_{1}^{2} w_{2}\right), \\
a_{12}=\frac{1}{\Delta}\left(\widetilde{\Delta}_{3} w_{1}+\widetilde{\Delta}_{4} w_{2}\right)=\frac{a_{1}^{2} b_{1} w_{1}}{\Delta}, \quad a_{21}=\frac{1}{\Delta}\left(\Delta_{1} w_{1}+\Delta_{2} w_{2}\right)=\frac{-a_{2} b_{2}^{2} w_{2}}{\Delta}, \\
a_{22}=\frac{1}{\Delta}\left(\Delta_{3} w_{1}+\Delta_{4} w_{2}\right)=\frac{1}{\Delta}\left(-a_{2}^{2} b_{2} w_{1}+\left(a_{2}^{2} b_{1}+a_{1} a_{2} b_{2}\right) w_{2}\right) .
\end{gathered}
$$

Then, it is easy to verify that

$$
w_{1}^{3}=a_{11} \tilde{P}_{1}+a_{12} \tilde{P}_{2}, \quad w_{2}^{3}=a_{21} \tilde{P}_{1}+a_{22} \tilde{P}_{2} .
$$

We calculate $\operatorname{det} A$ :

$$
\operatorname{det} A=\frac{1}{\Delta}\left(a_{2} b_{2} w_{1}^{2}-a_{2} b_{1} w_{1} w_{2}-a_{1} b_{1} w_{2}^{2}\right) .
$$

By Theorem 3

$$
\begin{gathered}
J_{(0,0)}=\sum_{\|K\| \leqslant 2} \frac{(-1)^{\|K\|} \cdot\left(k_{11}+k_{12}\right) ! \cdot\left(k_{21}+k_{22}\right) !}{k_{11} ! \cdot k_{12} ! \cdot k_{21} ! \cdot k_{22} !} \times \\
\times \mathfrak{M}\left[\frac{\tilde{\Delta} \cdot \operatorname{det} A \cdot \tilde{Q}_{1}^{k_{11}+k_{21}} \cdot \tilde{Q}_{2}^{k_{12}+k_{22}} \cdot a_{11}^{k_{11}} \cdot a_{12}^{k_{12}} \cdot a_{21}^{k_{21}} \cdot a_{22}^{k_{22}}}{w_{1}^{3\left(k_{11}+k_{12}\right)+1} \cdot w_{2}^{3\left(k_{21}+k_{22}\right)+1}}\right] . \\
-294-
\end{gathered}
$$


Denote $\bar{\Delta}=a_{2} b_{1}+a_{1} b_{2}$. Cumbersome but simple calculations (using the definition of the functional $\mathfrak{M}$ ) give that

$J_{(0,0)}=\frac{1}{\bar{\Delta}}-\frac{2 a_{1} b_{2}}{a_{2} b_{1} \bar{\Delta}}+\frac{6 a_{1}^{2} b_{2}^{2}}{a_{2} b_{1} \bar{\Delta}^{2}}-\frac{b_{2}^{3}}{b_{1} \bar{\Delta}^{2}}+\frac{a_{1}^{3}}{a_{2} \bar{\Delta}^{2}}+\frac{8 a_{1} b_{2}}{\bar{\Delta}^{2}}-\frac{4}{a_{2} b_{1}}=\frac{a_{1}^{3}}{a_{2} \bar{\Delta}^{2}}-\frac{a_{1} b_{2}}{\bar{\Delta}^{2}}-\frac{3 a_{2} b_{1}}{\bar{\Delta}^{2}}-\frac{b_{2}^{3}}{b_{1} \bar{\Delta}^{2}}$.

This work was supported by the Russian Science Foundation, grant Complex analytic geometry and multidimensional deductions. Number: 20-11-2011\%.

\section{References}

[1] L.A.Aizenberg, On a formula of the gereralized multidimensional logarithmic residue and the solution of system of nonlinear equations, Sov. Math. Doc., 18(1977), 691-695.

[2] L.A.Aizenberg, A.P.Yuzhakov, Integral representations and residues in multidimensional complex analysis, Trans. Amer. Math. Monographs, AMS, Providence, 1983.

[3] A.K.Tsikh, Multidimensional residues and their applications. Translations of Mathematical Monographs, 103. American Mathematical Society, Providence, RI, 1992.

[4] V.Bykov, A.Kytmanov, M.Lazman, M.Passare (ed), Elimination Methods in Polynomial Computer Algebra, Ser. 448 Math. and Appl., Kluwer Acad. Publ., Dordreht, Boston, London, 1998.

[5] L.A.Aizenberg, A.M.Kytmanov, Multidimensional analogues of Newton's formulas for systems of nonlinear algebraic equations and some of their applications, Siberian Mathematical Journal, 22(1981), 180-189.

[6] V.I.Bykov, Modeling of the critical phenomena in chemical kinetics, Komkniga, Moscow, 2006 (in Russian).

[7] V.I.Bykov, S.B.Tsybenova, Non-linear models of chemical kinetics, KRASAND, Moscow, 2011 (in Russian).

[8] A.M.Kytmanov, Z.E.Potapova, Formulas for determining power sums of roots of systems of meromorphic functions, Izvestiya VUZ. Matematika, 49(2005), no. 8, 36-45 (in Russian).

[9] V.I.Bykov, A.M.Kytmanov, S.G.Myslivets, Docl. Math., 76(2007), no. 2, 641-645. DOI: $10.1134 /$ S1064562407050018

[10] A.M.Kytmanov, E.K.Myshkina, Russian Mathematics, 57(2013), no. 12, 31-43. DOI: $10.3103 /$ S1066369X13120049

[11] A.M.Kytmanov, E.K.Myshkina, J. Math. Sciences, 213(2016), no. 6, 868-886. DOI: $10.1007 / \mathrm{s} 10958-016-2748-7$

[12] A.A.Kytmanov, A.M.Kytmanov, E.K.Myskina, Finding residue integrals for systems of nonalgebraic equations in $\mathbb{C}^{n}$, Journal of Symbolic Computation, 66(2015), 98-110.

[13] A.M.Kytmanov, O.V.Khodos, On systems of non-algebraic equation in $\mathbb{C}^{n}$, Contemporary Mathematics, 662(2016), 77-88.

[14] A.M.Kytmanov, E.K.Myshkina, On calculation of power sums of roots for one class of systems of non-algebraic equations, Sib. Elektron. Mat. Izv., 12(2015), 190-209 (in Russian). DOI: https://doi.org/10.17377/semi.2015.12.016 
[15] O.V.Khodos, On Some Systems of Non-algebraic Equations in $\mathbb{C}^{n}$, Journal of Siberian Federal University. Mathematics \&Physics, 7(2014), no. 4, 455-465.

[16] A.M.Kytmanov, E.R.Myshkina, Russian Mathematics, (2019), no. 5, 40-55. DOI: $10.26907 / 0021-3446-2019-5-40-55$

[17] A.M.Kytmanov, Algebraic and transcendental systems of equations, SFU, Krasnoyarsk, 2019.

[18] M.Passare, A.Tsikh, Residue integrals and their Mellin transforms, Can. J. Math., 47(1995), no. $5,1037-1050$.

[19] A.M.Kytmanov, A transformation formula for Grothendieck residues and some of its applications, Siberian Mathematical Journal, 29(1988), no. 3, 495-499.

[20] V.R.Kulikov, V.A.Stepanenko, On solutions and Waring's formulae for the system of $\mathrm{n}$ algebraic equations with n unknowns, St. Petersburg Math. J., 26(2015), no. 5, 839-848.

\title{
О некоторых примерах систем трансцендентных уравнений
}

\section{Александр М. Кытманов \\ Ольга В. Ходос}

Сибирский федеральный университет

Российская Федерация

\begin{abstract}
Аннотация. В данной статье рассматриваются примеры трансцендентных систем уравнений общего вида. Интегралы вычетов определяются по циклам, связанным с системой. Приведены формулы для их расчета, и установлена связь со степенными суммами корней системы.
\end{abstract}

Ключевые слова: трансцендентные системы уравнений, интегралы вычетов, степенные суммы корней. 